\title{
A genetic register for von Hippel-Lindau disease
}

I R Maddock, A Moran, E R Maher, M D Teare, A Norman, S J Payne, R Whitehouse, C Dodd, M Lavin, N Hartley, M Super, D G R Evans

\section{Abstract}

A genetic register for von Hippel-Lindau disease was set up in the north west of England in 1990. Population statistics, clinical features, age at onset, and survival of 83 people affected with von Hippel-Lindau (VHL) disease were studied. In addition, the effectiveness of the screening programme used and the occurrence of central nervous system haemangioblastomas in the general population were examined. The diagnostic point prevalence of heterozygotes in the North Western Region was $1 \cdot 18 / 100000$ (1/ 85000 ) people, with an estimated birth incidence of $2 \cdot 20 / 100000(1 / 45500)$ live births. The mutation rate was estimated directly to be $1 \cdot 4 \times 10^{-6} /$ gene/generation $(1 / 714200)$.

The mean age at onset of first symptoms was $\mathbf{2 6 \cdot 2 5}$ years, with cerebellar haemangioblastoma being the most common presenting manifestation ( $34.9 \%$ of cases). The mean age at diagnosis of VHL disease was 30.87 years. Overall, 50 patients $(60 \cdot 2 \%)$ developed a cerebellar haemangioblastoma, $34(41.0 \%)$ a retinal angioma, $21(25 \cdot 3 \%)$ a renal cell carcinoma, $12(14 \cdot 5 \%)$ a spinal haemangioblastoma, and $12(14.5 \%)$ a phaeochromocytoma.

Mean age at diagnosis of renal cell carcinoma (38.9 years) was significantly higher than that for cerebellar haemangioblastoma (30.0 years) and retinal angioma (21.1 years). Mean age at death was 40.9 years with cerebellar haemangioblastoma being the most common cause ( $47 \cdot 7 \%$ of deaths).

A total of 65 VHL manifestations were diagnosed asymptomatically following appropriate clinical and radiological screening tests, and failure to detect manifestations of VHL disease in spite of appropriate screening occurred on only two occasions. The use of DNA linkage analysis and direct mutation testing reduced the personal risk of carrying the VHL gene to below $1 \%$ in 14 people. In addition to the 83 clinically affected subjects, three obligate carriers who were considered to be lesion free in spite of extensive screening tests were identified.

Fourteen percent of all CNS haemangioblastomas on the regionally based Cancer Registry were found to occur as part of VHL disease, but investigations for VHL in apparently sporadic disease appeared to be limited.

(f Med Genet 1996;33:120-127)

Key words: von Hippel-Lindau disease; genetic register.
Von Hippel-Lindau (VHL) disease is an autosomal dominant, familial cancer syndrome which most commonly affects the central nervous system (CNS), eyes, kidneys, adrenal glands, and pancreas. ${ }^{1-3}$ The most frequent complications include haemangioblastomas (HB) of the CNS and retina, renal cell carcinoma (RCC), phaeochromocytoma, and pancreatic malignancy, as well as renal and pancreatic cysts. It is a potentially devastating disease and its successful management is a challenge to specialists in general surgery, genetics, haematology, medicine, nephrology, neurology, neurosurgery, oncology, ophthalmology, pathology, paediatrics, and uro$\operatorname{logy} .^{2}$ There are no strict diagnostic criteria for VHL. However, in 1990, after a large population based study Maher et $a l^{\beta}$ stated that the diagnosis of VHL could be made if multiple retinal or cerebellar haemangioblastomas were diagnosed in the same person, or if a single retinal or cerebellar haemangioblastoma was accompanied by a renal carcinoma or phaeochromocytoma. In addition, VHL can be diagnosed in the presence of a single haemangioblastic or visceral tumour if there is a positive family history. ${ }^{4}$

In 1988, Seizinger $e t a l^{5}$ mapped the gene for VHL disease to the short arm of chromosome 3 , close to the RAF1 oncogene at $3 p 25$, by genetic linkage analysis. Close flanking DNA markers had been identified which made presymptomatic diagnosis of VHL disease possible $^{6}$; additional close flanking DNA markers were identified in $1993 .^{7}$ The VHL gene was eventually cloned in May $1993,{ }^{8}$ making presymptomatic diagnosis of VHL patients possible with mutation analysis.

Although VHL is not a common disease, it can be devastating both to affected subjects and the rest of their family. Each of the manifestations of VHL disease poses a serious threat to the health of affected subjects, but if they are detected at an early stage they are all treatable. ${ }^{2}$ Hence, a screening programme to detect further tissue involvement in people known to have VHL disease and to identify new cases in the affected person's family is an essential part of the management of both the patient and his/her relatives. ${ }^{1-4-13}$ A regular clinical examination, with particular attention to the CNS and abdomen, will identify a large proportion of lesions associated with VHL disease. In addition, specific screening tests are available for all the serious manifestations of the disease. Ocular involvement may be monitored by regular indirect ophthalmoscopy/ fluorescein angioscopy or angiography, ${ }^{24}$ and 
involvement of the abdominal organs may be detected safely and relatively inexpensively using US scanning. Involvement of the CNS and abdominal organs may also be detected using CT or MR scanning. MR scanning has the advantages of being more sensitive and posing less of a radiation risk than CT scanning (which may result, for example, in radiation induced cataract). ${ }^{10}$ In addition, analysis of a 24 hour urine specimen for a high level of VMAs and normetadrenaline may also detect phaeochromocytomas.

As part of our extensive genetic register in the north west of England we have set up a register for VHL incorporating the screening programme proposed by Maher et $a^{\beta}$ in 1990 . The identification of linked DNA markers during the past few years and the more recent identification of VHL gene mutations has allowed accurate presymptomatic diagnosis of the disease in many cases. ${ }^{36714-16}$ We report here the epidemiological data from our regional VHL disease register and assess the effectiveness of the screening programme which has been set up within the North West region.

\section{Patients and methods}

A register for people affected with VHL disease was set up in the North West Regional Genetics Centre at St Mary's Hospital, Manchester, in 1990. Information was obtained retrospectively from the hospital notes of 23 families who were known to have at least one family member who had been diagnosed as having VHL disease (as defined earlier). This included 21 families who were being followed up at St Mary's Hospital, Manchester, and were therefore included on the VHL disease register, and two families who were being followed up at the Royal Manchester Children's Hospital. Information was also obtained retrospectively from the hospital notes of a further five families who had been referred to St Mary's Hospital for investigations following the diagnosis of a single VHL disease manifestation in one family member. Since these families were only suspected of having VHL disease, they have been analysed separately. Details of patients who had died were obtained from hospital notes and necropsy reports when available.

The information obtained was used to complete a detailed proforma for each person in each of the 28 families, and each proforma was used to transfer the information on to a computer database for analysis. On the study day (30 November 1994), details concerning 934 people from families known to be affected with VHL disease, and 132 people from families suspected of being affected with VHL disease were available for analysis. The prevalence of heterozygotes was calculated based on the population living within the area served by the North Western Regional Health Authority, since ascertainment in this population was likely to be highest. The number of heterozygotes in the region was estimated by combining: (1) the number of affected people living in the region; (2) the number of asymptomatic heterozygotes who were likely to be among the relatives at $50 \%$ prior (or empirical) risk of inheriting the disease within the region. The age related risk for unaffected subjects at $50 \%$ prior risk was calculated using the age at onset data obtained by Maher et $a^{17}$ in 1991; (3) the number of isolated cases below the age of 25 years. Below this age, most isolated cases will not have been diagnosed, ${ }^{17}$ and hence the number of cases was estimated by adjusting the number of isolated cases aged 25 to 49 years to take into account the difference between the populations of these two age groups (population of NW aged $0-24$ years $=1361283$; population of NW aged $25-49=1428471) .^{18}$

The total number of heterozygotes living in the north west was then used to calculate the point prevalence of the disease (based on a total population of the NW region of 4030800$).{ }^{18}$

The diagnostic prevalence underestimates the birth incidence because of the late age at presentation and early age at death. Therefore, the birth incidence for VHL disease in the NW region was calculated by determining the number of living heterozygotes aged 25 to 49 years (the age group in which ascertainment of heterozygotes is likely to be most accurate), taking into account both the known heterozygotes and those contained within the 50\% prior risk group. After the addition of further subjects with VHL disease born between 30.11.1944 and 1.12.1969 but who had died before the study date, the total was divided by the total number of people in the NW region aged 25-49 years to obtain the prevalence in this age group; this figure was taken to be an estimate of the birth incidence.

In order to estimate the mutation rate, the number of new mutations (as determined by pedigree analysis) in those aged 25-49 years was divided by the population of this age group in the NW region to give the population frequency of new mutations in this age group. This was then halved to give a direct estimate of the mutation rate.

The screening programme used for all affected and at risk subjects is summarised in the appendix. All feature diagnoses made were divided by the number of screens made and any interval diagnosis was recorded.

The identification of cases of VHL disease that had not been referred to the genetic departments, and hence were not included on the VHL disease register, was attempted using the North West Cancer Registry. A listing was produced of all central nervous system HBs registered between 1971 and 1993. The list of cases was matched against the VHL register. Efforts were made to identify patients with retinal angioma by searching patient records at the local regional ophthalmological referral centre. The registry database was searched for the occurrence of a second primary neoplasm in the people with CNS HBs. Hospital notes were requested for those people in whom a second primary neoplasm had been diagnosed, and also for patients who were 35 years of age or younger at the time of diagnosis of the HB. The information obtained from the cancer registry was also used to estimate the percentage of all CNS HBs that occur as part of 
Table 1 Presenting manifestations of 83 subjects with VHL disease

\begin{tabular}{lcc}
\hline Presenting manifestation & No of subjects & $\%$ \\
\hline Retinal angioma & 21 & $25 \cdot 3$ \\
Cerebellar haemangioblastoma & 29 & $34 \cdot 9$ \\
Spinal haemangioblastoma & 2 & $2 \cdot 4$ \\
Renal cell carcinoma & 7 & $8 \cdot 4$ \\
Phaeochromocytoma & 6 & $7 \cdot 2$ \\
Pancreatic carcinoma & 2 & $2 \cdot 4$ \\
Meningioma & 1 & $1 \cdot 2$ \\
Pituitary haemangioblastoma & 1 & $1 \cdot 2$ \\
Duodenal carcinoid tumour & 1 & $1 \cdot 2$ \\
Asymptomatic & 6 & $7 \cdot 2$ \\
Not known & 7 & $8 \cdot 4$ \\
\hline
\end{tabular}

Table 2 Frequency of manifestations of VHL disease

\begin{tabular}{lccc}
\hline Manifestation & $\begin{array}{l}\text { No of subjects } \\
\text { affected (\%) }\end{array}$ & $\begin{array}{l}\text { No in whom } \\
\text { manifestation } \\
\text { occurred more } \\
\text { than once (\%) }\end{array}$ & $\begin{array}{l}\text { Total no of } \\
\text { diagnoses } \\
\text { made }\end{array}$ \\
\hline Retinal angioma & $34(41 \cdot 0)$ & $6(17 \cdot 6)$ & 44 \\
Cerebellar haemangioblastoma & $50(60 \cdot 2)$ & $17(34 \cdot 0)$ & 77 \\
Brainstem haemangioblastoma & $3(3 \cdot 6)$ & $1(33 \cdot 3)$ & 4 \\
Spinal haemangioblastoma & $12(14.5)$ & $0(0)$ & 12 \\
Renal cell carcinoma & $21(25 \cdot 3)$ & $3(14 \cdot 3)$ & 24 \\
Phaeochromocytoma & $12(14 \cdot 5)$ & $1(8 \cdot 3)$ & 13 \\
Renal cysts & $20(24 \cdot 1)$ & $3(15 \cdot 0)$ & 24 \\
Pancreatic cysts & $13(15 \cdot 7)$ & $0(0)$ & 13 \\
Pancreatic carcinoma & $4(4 \cdot 8)$ & $0(0)$ & 4 \\
Pancreatic apudoma & $1(1 \cdot 2)$ & $0(0)$ & 1 \\
Meningioma & $2(2 \cdot 4)$ & $0(0)$ & 2 \\
Hepatic cysts & $2(2 \cdot 4)$ & $0(0)$ & 1 \\
Polycythaemia & $1(1 \cdot 2)$ & $0(0)$ & 1 \\
Pituitary haemangioblastoma & $1(1 \cdot 2)$ & $0(0)$ & 1 \\
Duodenal carcinoid tumour & $1(1 \cdot 2)$ & $0(0)$ & \\
\hline
\end{tabular}

Table 3 Mean age at initial diagnosis of each of the manifestations of VHL disease

\begin{tabular}{llccr}
\hline Manifestation & Mean $(y)$ & $S D(y)$ & Range $(y)$ & No* \\
\hline Retinal angioma & $21 \cdot 1$ & $9 \cdot 11$ & $8-49$ & 29 \\
Cerebellar haemangioblastoma & $30 \cdot 0$ & $11 \cdot 92$ & $15-56$ & 43 \\
Brainstem haemangioblastoma & $24 \cdot 3$ & $4 \cdot 51$ & $20-29$ & 3 \\
Spinal haemangioblastoma & $37 \cdot 1$ & $11 \cdot 76$ & $23-58$ & 10 \\
Renal cell carcinoma & $38 \cdot 9$ & $10 \cdot 46$ & $25-58$ & 16 \\
Phaeochromocytoma & $25 \cdot 9$ & $16 \cdot 92$ & $12-58$ & 10 \\
Renal cysts & $38 \cdot 8$ & $13 \cdot 17$ & $24-64$ & 19 \\
Pancreatic cysts & $30 \cdot 0$ & $6 \cdot 10$ & $24-44$ & 13 \\
Pancreatic carcinoma & $49 \cdot 0$ & $13 \cdot 75$ & $34-61$ & 3 \\
Pancreatic apudoma & $41 \cdot 0$ & - & - & 1 \\
Meningioma & $32 \cdot 0$ & $21 \cdot 21$ & $17-47$ & 2 \\
Hepatic cysts & $30 \cdot 0$ & $8 \cdot 49$ & $24-36$ & 2 \\
Polycythaemia & NK & - & - & -1 \\
Pituitary haemangioblastoma & $20 \cdot 0$ & - & - & 1 \\
Duodenal carcinoid tumour & $15 \cdot 0$ & - & - & \\
\hline
\end{tabular}

* In some cases, age at initial diagnosis of the manifestation was not known.

VHL disease, and to examine the distribution of age at onset of CNS HBs.

Statistical analysis was performed using unpaired $t$ testing, with statistical significance taken to be $<5 \%$ (or $\mathrm{p}<0.05$ ).

\section{Results}

FAMILIES WITH PROVEN VHL DISEASE

Of the 934 people from 23 families identified as having VHL, 432 were male, 429 were female, and in 73 the sex was not known. A total of 692 people were alive on the study day, with ages ranging from 5 months to 93 years; 83 affected subjects were identified, of whom 13 were considered to be new mutations. There were 49 subjects at $50 \%$ risk, 51 at $25 \%$ risk, and 18 parents and sibs of apparent new mutations resident in the North Western Region.

\section{PREVALENCE}

Of the 83 affected subjects, 54 were resident in the NW region, and 26 of these were alive on the study day. The 49 subjects alive in the NW region who had a prior risk of $50 \%$, when analysed using the age related risk curve, showed that there were likely to be $17 \cdot 6$ heterozygotes among them. The number of isolated cases aged 25-49 years was found to be four (including two isolated cases born between 1.12.1944 and 30.11.1969 who had died before the study day), and from this it was estimated that there were 3.8 isolated heterozygotes under the age of 25 years in the NW region who have not yet been diagnosed.

Therefore, the total number of heterozygotes for VHL disease in the NW region was estimated to be $47 \cdot 4$, giving a minimum heterozygote prevalence of 1.18 per 100000 or 1 per 85000 people.

\section{BIRTH INCIDENCE}

The number of affected subjects who were between the ages of 25 and 49 years and who were living in the NW region was found to be 17. There were also 26 subjects in the same age group and living in the NW region who were at a $50 \%$ prior risk of carrying the VHL disease gene; analysis using the age related risk curve showed that there were likely to be $4 \cdot 4$ heterozygotes among them. In addition, there were 10 affected subjects who were born between 1.12.1944 and 30.11.1969 but who had died before the study day. Therefore, the total number of heterozygotes for VHL disease in the 25-49 year age group was estimated to be 31.4 , giving a minimum birth incidence (which was taken to be the prevalence in this age group) of $2 \cdot 20$ per 100000 or 1 per 45500 live births.

\section{MUTATION RATE}

In the NW region, four patients aged between 25 and 49 years (including two cases born between 1.12.1944 and 30.11.1969 who had died before the study day) were considered to be new mutations, giving a population frequency of new mutations of $0 \cdot 28$ per 100000 or 1 per 357100 people. Hence, the mutation rate (estimated directly) was $0 \cdot 14$ per 100000 or 1 per 714200 genes.

\section{FIRST MANIFESTATION OF VHL DISEASE}

The presenting manifestations of VHL disease are shown in table 1 . Five of the clinically healthy people were detected by radiological screening tests, and one affected subject was detected using DNA linkage analysis. The mean age at onset of first symptom was $26 \cdot 25$ $(\mathrm{SD}=12 \cdot 53$, range $=10-62)$ years $(\mathrm{n}=64)$, and the mean age at diagnosis of VHL disease was $30 \cdot 87(\mathrm{SD}=14 \cdot 36$, range $=8-62)$ years $(\mathrm{n}=71)$. The proportion of patients who had presented by the ages of 30,40,50, and 60 years was $60 \cdot 6 \%, 69 \cdot 0 \%, 83 \cdot 1 \%$, and $98.6 \%$, respectively. The effect of excluding patients who had been detected by clinical or DNA screening $(n=6)$ on the age at diagnosis curve 
Table 4 VHL disease manifestations diagnosed asymptomatically

\begin{tabular}{lll}
\hline Manifestation & $\begin{array}{l}\text { Total no of } \\
\text { diagnoses made }\end{array}$ & $\begin{array}{l}\text { No of diagnoses made } \\
\text { presymptomatically (\%) }\end{array}$ \\
\hline Retinal angioma & 44 & $13(29 \cdot 5)$ \\
Cerebellar haemangioblastoma & 77 & $7(9 \cdot 1)$ \\
Brainstem haemangioblastoma & 4 & $1(25 \cdot 0)$ \\
Spinal haemangioblastoma & 12 & $7(25 \cdot 0)$ \\
Renal cell carcinoma & 24 & $3(23 \cdot 1)$ \\
Phaeochromocytoma & 13 & $17(70 \cdot 8)$ \\
Renal cysts & 24 & $12(92 \cdot 3)$ \\
Pancreatic cysts & 13 & $2(100)$ \\
Hepatic cysts & 2 &
\end{tabular}

was negligible, with the proportions of VHL patients who were symptomatic at diagnosis and who had presented by the ages of 30,40 , 50 , and 60 years being $60.0 \%, 69 \cdot 2 \%, 83 \cdot 1 \%$, and $98.5 \%$ respectively. There were no significant differences between the mean ages at diagnosis in symptomatic and asymptomatic cases, male and female cases, or familial and isolated cases $(p>0 \cdot 2)$. Table 2 shows the frequency of the manifestations of VHL disease found in our series of 83 affected subjects; the number who were diagnosed as having the same lesion on more than one occasion is also shown in each case. Three people were identified as being obligate carriers of the VHL gene. The mean age at initial diagnosis of each of the manifestations is shown in table 3.

\section{SCREENING}

Screening tests were undertaken in 104 subjects, 87 of whom were screened in the NW region. Sixty nine were investigated by CT head scanning, 70 by abdominal US or CT scanning, 78 by eye examination, and 78 by clinical examination and urine VMA analysis; 66 people underwent all four screening tests. A total of $29 / 42$ eligible people at $50 \%$ risk were screened (seven were under 5 years), as well as $15 / 18$ of the parents and sibs of apparent isolated cases. A total of $65 \mathrm{VHL}$ manifestations were diagnosed asymptomatically following ap- propriate screening tests (table 4 ). The numbers of diagnoses made in people who were screened within the NW region following initial (prevalence) screening and subsequent (follow up) screening tests are shown in table 5. For those screened in the NW region, the total numbers of screening years for CT head scan, abdominal US/CT scan, eye examination, and clinical examination were $78,129,171$, and 135 years respectively; these were used to calculate the numbers of diagnoses made per screening year, which are also shown in table 6. Screening tests failed to detect only two manifestations of VHL disease in the NW region; a CHB and a pancreatic carcinoma were diagnosed in two separate people following the onset of symptoms, despite their involvement in the regional screening programme at the time.

\section{MOLECULAR GENETIC ANALYSIS}

In 14 of 23 families the family structure was suitable for genetic linkage studies. At risk subjects from seven of these families requested presymptomatic DNA testing. Linkage analysis was informative in five families, uninformative in one family, and is in progress in one family. To date eight subjects with a prior risk of $50 \%$ and six with a prior risk of $25 \%$ have been shown to be at low risk $(<1 \%)$ by linkage analysis. Screening for VHL gene mutations has been performed in all 23 families and a germline mutation identified in $\mathbf{1 7}$ families containing 19 subjects at $50 \%$ prior risk within the North West Region. Three of these have undergone direct mutation analysis and were shown not to be gene carriers (one had been found to be low risk on linkage analysis). The remaining at risk people from the 17 families are in the process of being offered DNA testing. In one of these families the parents and two sibs of a presumed new mutation have been

Table 5 Numbers of diagnoses made following screening for VHL disease manifestations within the NW region

\begin{tabular}{lllll}
\hline Manifestation & $\begin{array}{l}\text { Diagnoses } \\
\text { following initial } \\
\text { tests }\end{array}$ & $\begin{array}{l}\text { No of diagnoses } \\
\text { per initial screen }\end{array}$ & $\begin{array}{l}\text { Diagnoses } \\
\text { following } \\
\text { subsequent tests }\end{array}$ & $\begin{array}{l}\text { No of diagnoses } \\
\text { per screening year }\end{array}$ \\
\hline Retinal angioma & 2 & 0.026 & 8 & 0.047 \\
Cerebellar haemangioblastoma & 6 & $0 \cdot 087$ & 1 & $0 \cdot 077$ \\
Brainstem haemangioblastoma & 0 & 0 & 1 & $0 \cdot 013$ \\
Spinal haemangioblastoma & 1 & 0.014 & 2 & $0 \cdot 016$ \\
Renal cell carcinoma & 1 & 0.014 & 0 & 0.039 \\
Phaeochromocytoma & 2 & $0 \cdot 029$ & 9 & 0 \\
Renal cysts & 7 & $0 \cdot 100$ & 4 & $0 \cdot 070$ \\
Pancreatic cysts & 8 & 0.114 & 0 & 0.031 \\
Hepatic cysts & 2 & 0.029 & & 0 \\
\hline
\end{tabular}

Table 6 Second primary neoplasms diagnosed in people with a CNS haemangioblastoma

\begin{tabular}{|c|c|c|c|}
\hline Second primary neoplasm & $\begin{array}{l}\text { Age at diagnosis of } 2 \text { nd } \\
\text { primary neoplasm }\end{array}$ & $\begin{array}{l}\text { CNS HB in these } \\
\text { subjects }\end{array}$ & $\begin{array}{l}\text { Age at diagnosis of CNS } \\
\mathrm{HB}\end{array}$ \\
\hline $\begin{array}{l}\text { Pancreatic carcinoma* } \\
\text { Uterine adenocarcinoma } \\
\text { Uterine squamous cell carcinoma } \\
\text { Infiltrating ductal breast } \\
\text { Basal cell carcinoma } \\
\text { Chronic lymphocytic leukaemia } \\
\text { Bronchogenic carcinoma } \\
\text { Renal cell carcinomat } \\
\text { Renal cell carcinomat } \\
\text { Not known }\end{array}$ & $\begin{array}{l}64 \\
53 \\
25 \\
67 \\
59 \\
57 \\
62 \\
39 \\
38 \\
57\end{array}$ & $\begin{array}{l}\text { CHB } \\
\text { CHB } \\
\text { CHB } \\
\text { CHB } \\
\text { CHB } \\
\text { CHB } \\
\text { CHB } \\
\text { CHB } \\
\text { SHB } \\
\text { CHB }\end{array}$ & $\begin{array}{l}48 \\
53 \\
25 \\
63 \\
59 \\
71 \\
70 \\
22 \\
29 \\
72\end{array}$ \\
\hline
\end{tabular}

* This person may well have had VHL disease.

† These people are known to have VHL disease. 
shown not to carry the gene defect. One of the families in which a VHL mutation was not identified has a suitable structure for linkage analysis and informative testing is in progress. Only two subjects have positively declined predictive testing, one at $50 \%$ risk and the other being the brother of a presumed new mutation; both have partaken in clinical and radiological screening. It is anticipated that the majority of people undergoing active screening will undertake predictive testing.

\section{AGE AT DEATH OF VHL PATIENTS}

Of the 83 affected subjects, 44 had died by the study day, and information regarding age at death was available for 39 of these. Mean age at death in VHL patients was found to be 40.9 $(\mathrm{SD}=14 \cdot 57$, range $=12-65)$ years $(n=39)$. The most common cause of death was $\mathrm{CHB}$, accounting for $21(47 \cdot 7 \%)$ of the deaths from VHL disease. Twelve deaths (27.3\%) were caused by RCCs, three $(6 \cdot 8 \%)$ by pancreatic carcinomas, two $(4.5 \%)$ by phaeochromocytomas, one $(2 \cdot 3 \%)$ by a BSHB, and one $(2 \cdot 3 \%)$ by a SHB. In four affected subjects, the cause of death was not known.

REFERRAL OF AFFECTED FAMILIES

Seven out of the 23 VHL families were referred by GPs, five by ophthalmologists, and three each by neurosurgeons and neurologists.

CNS HAEMANGIOBLASTOMAS AND VHL DISEASE Using the Regional Cancer Registry, a total of 86 people were identified as having a CNS HB diagnosed between 1971 and 1993. Eleven of these were on the VHL genetic register, while none of the remainder had been seen in the eye hospital. Ten of the subjects diagnosed with a CNS HB were found to have another tumour on the Registry database (table 6). The two patients with renal cell carcinoma were known to have VHL. In addition, 21 of the 83 affected subjects with VHL in our series, who were living in the registry area, had a CNS HB diagnosed between 1971 and 1993, but only 11 $(53 \%)$ of them were registered on the Regional Cancer Registry. However, one was operated on outside the region and three were detected on CT scans in known affected VHL subjects who have not undergone surgery. A further case also had a registration for renal cell carcinoma.

The eight cases with a second primary, not known to have VHL and 19/29 cases under 35 years who were also known to the genetic register had their medical records requested; $21 / 26$ sets of notes were available for scrutiny (one case of double primary occurred <35 years, five had been destroyed). The diagnosis of CNS HB was confirmed in each case as was the second primary in $5 / 6$ (the lung cancer diagnosis was incorrect). The person with the most likely second primary to be part of VHL had undergone laparotomy a few weeks before death and also had a necropsy; however, histology was not requested. Nonetheless, no other disease features apart from the pancreatic tumour were found. Among the 21 sets of notes there were no definite confirmed cases of VHL, although one subject suffered two recurrences of the original cerebellar HB and developed a spinal HB nine years after initial diagnosis and this almost certainly represents VHL. ${ }^{3}$

The possibility of VHL was mentioned in only 4/21 cases: once in the pathology report, once by a referring physician, and only twice by the treating neurosurgeon. In the latter two cases limited further investigations were undertaken. In a number of other cases VHL appeared to have been considered because of the investigations carried out. Several cases had been referred for ophthalmic examination and one had had a normal abdominal ultrasound scan. Three asymptomatic sibs of one case had brain scans though the affected subject did not have any further investigation.

\section{Discussion}

POPULATION STATISTICS

Since VHL disease was identified as a distinct disease entity in 1926, there have been more than 700 cases reported in English language publications. ${ }^{23}$ To the best estimate of the various ascertainment methods used the diagnostic prevalence of heterozygotes for VHL disease in the NW region ( $1 / 85000$ people) is of the same order of magnitude as that estimated in previous reports, but when compared with some recent reports, ${ }^{17} 19$ it is considerably lower, although Maher et $a l^{17}$ concede that their estimate of the point prevalence in East Anglia ( $1 / 53000$ people) is somewhat higher than in other reports. The birth incidence of VHL disease (1/45 500 live births), although also being a little lower than the one estimated by Maher et $a l,{ }^{17}$ is very similar to the one calculated by Harries. ${ }^{9}$

\section{FIRST MANIFESTATION OF VHL DISEASE}

CHB was the most common presenting manifestation of VHL disease, with RA (which is regarded by most as being the earliest manifestation of VHL disease) ${ }^{3}$ being the second most common in our series. However, the mean ages at onset and at diagnosis of RA when it was the presenting complication were considerably lower than those of CHB. Mean ages at onset overall and for each of the four most common presenting manifestations were similar to those found in other similar studies. ${ }^{23}$ However, we found that on average the diagnosis of VHL disease was made over four and a half years after the onset of symptoms. Again, screening and higher awareness of the disease may help to reduce this delay. Although most subjects show features of VHL disease relatively early on in life, the diagnosis of the disease was made in 12 people who were over the age of 50 years and in one person who was over the age of 60 years. Hence, screening of at risk subjects should continue at least until the age of 65 years if cases of VHL disease are not to be overlooked. 
MANIFESTATIONS OF VHL DISEASE

CHB was the most common complication of VHL disease, followed in order by RA, RCC, renal cysts, pancreatic cysts, phaeochromocytomas, and SHBs. The percentages of subjects who were affected with each of the common manifestations are similar to those quoted in other reports ${ }^{23}$ with the exception of RA with which considerably fewer people were affected. It is also generally accepted that pancreatic carcinoma in VHL disease is not as common as pancreatic apudoma, ${ }^{2}$ but this was not the case in our series (although numbers were small); pancreatic carcinoma was four times more common and was the presenting manifestation in two cases. Several relatively rare tumours were diagnosed in affected subjects. Two cases of meningioma were identified, which may have been part of the syndrome or may have been coincidental findings. Polycythaemia was diagnosed in one person, but this was not associated with either $\mathrm{CHB}$ or RCC, and hence may also have been a coincidental finding. Hepatic cysts were identified in two people, but since these are rare in the general population ${ }^{2}$ it is likely that they were manifestations of the disease. A pituitary haemangioblastoma was diagnosed in one person as being part of the VHL syndrome, as was a duodenal carcinoid tumour; although they have not been reported as complications of VHL disease, these lesions are otherwise so rare that it is quite likely that they have occurred as part of the syndrome. The mean age at initial diagnosis of the VHL disease manifestations are comparable with those found in other studies. ${ }^{23}$ The late age at onset and at diagnosis of RCC contributes greatly to its high mortality.

\section{SCREENING}

Clinical and radiological

Of the common VHL disease manifestations, the percentage of those detected by screening tests was highest for pancreatic cysts, followed in order by renal cysts, RAs, RCCs, SHBs, BSHBs, phaeochromocytomas, and CHBs. However, the high rate of detection in the first two lesions on this list is because the majority of these lesions are asymptomatic. Although early detection and treatment of, for example, RA or RCC is beneficial, a CHB is usually only treated if the patient is symptomatic; hence, failure to detect a CHB will not greatly influence the mortality or morbidity associated with the condition. However, the diagnosis of an asymptomatic CHB in a previously unaffected at risk subject establishes that this person is a gene carrier, and allows optimal follow up care, and thus it remains important that these lesions are detected. There were no statistically significant differences between the ages at diagnosis of VHL manifestations in symptomatic cases and those detected asymptomatically. However, asymptomatic cases were diagnosed at an earlier age than symptomatic cases in CHB, RCC, pancreatic cysts, and renal cysts, although this is probably because these lesions usually remain asymptomatic for a number of years before symptoms eventually occur. Once the VHL register has been longer established, it is likely that the majority of lesions will be detected asymptomatically. The rates of detection of asymptomatic lesions (table 5) also show that the highest detection rates are found in those lesions that often remain asymptomatic: $\mathrm{CHB}$, pancreatic cysts, and renal cysts. The occurrence of a $\mathrm{CHB}$ and a pancreatic carcinoma as interval tumours shows that the sensitivity of the screening tests is not $100 \%$, but this is to be expected since the attainment of $100 \%$ sensitivity would mean overinvestigation in the majority of cases. However, the use of MR scanning in place of $\mathrm{CT}$ scanning may reduce the number of lesions that pass undetected.

\section{DNA TESTING}

In our series, DNA linkage analysis and direct mutation testing proved useful in confirming the diagnosis of VHL disease in six families and in substantially reducing the probability of carrying the VHL disease gene in a further 18 subjects; in the latter it was considered appropriate to relax the screening programme. In future years, with improved techniques of identification of mutations, it will hopefully be possible to offer predictive testing to the great majority of at risk people. Although screening using radiological and genetic tests may reduce the morbidity and mortality associated with VHL disease, it must be remembered that long term compliance with the complicated screening protocol may be difficult to achieve. Nonetheless, gene testing will concentrate screening on those really at risk and the resources saved on unaffected subjects may allow more rigorous screening of the gene carriers.

\section{DEATH FROM VHL DISEASE}

The mean age at death was similar to that found in other studies. ${ }^{23}$ The majority of previous reports have found $\mathrm{CHB}$ to be the most frequent cause of death in VHL disease patients, ${ }^{20}$ and this was also the case in our study. However, one must also take into account that nearly two and a half times as many people were diagnosed with a $\mathrm{CHB}$ than were diagnosed with a RCC, and hence RCC (when present) was the lesion with the highest mortality. This is further substantiated by the values for survival following treatment, which show that the lowest duration of survival was found following nephrectomy for RCC.

OBLIGATE CARRIERS OF THE VHL DISEASE GENE Three subjects remained asymptomatic in spite of having both affected sibs and children, and were hence identified as being obligate carriers of the VHL disease gene. ${ }^{21}$ Two of these are alive and aged 53 years and 67 years, while the third died of pneumonia in 1990 at the age of 50 years. The 67 year old subject has previously been reported, ${ }^{18}$ and was only recently found after careful screening to have bilateral renal cysts. Although commonly found as part of VHL disease (with one study reporting that 
they occur in more than two thirds of VHL patients), ${ }^{9}$ renal cysts are also a common finding in the general population. One series found that $27 \%$ of the general population over the age of 50 years were found to have renal cysts on CT scanning ${ }^{22}$ and hence the finding of renal cysts in this 67 year old subject may simply reflect an age related variant of normal. The occurrence of obligate carriers in VHL disease has obvious implications for screening protocols in that it would appear to be appropriate to continue with screening beyond the age of 60 years in order to identify the small number of affected people who may present later in life, particularly as this presentation is likely to be with a renal cell carcinoma.

CNS HAEMANGIOBLASTOMAS AND VHL DISEASE When comparing the CNS HBs identified as part of VHL disease with those identified using the Regional Cancer Registry, the former were found to have a lower mean age at diagnosis. In addition, the percentage of CHBs diagnosed by the age of 35 years was $90.7 \%$ in VHL patients and $24.3 \%$ in the general population. This is consistent with the view that CNS HBs occur at a much earlier age in VHL disease than as a solitary occurrence in the general population. Our estimate of the percentage of CHBs which occur as part of VHL disease is low when compared with others. ${ }^{12}$ This may reflect the very low level of suspicion for the diagnosis and the few patients that have had specific investigations for VHL. A few cases may have been diagnosed with other manifestations of VHL and were not identified by this study if these were diagnosed in a different hospital and the manifestation was not registrable with the cancer registry. Forty seven percent of the VHL HBs had not been registered with the Regional Cancer Registry although this may partly have been because of screen only detection with no surgery. Sporadic tumours with $\mathrm{HB}$ as the primary diagnosis would be less likely to have missed registration.

\section{ROLE OF THE GENETIC REGISTER FOR VHL}

DISEASE

The role of the clinical geneticist is not only to counsel and screen people who are referred to the register with a specific condition, but it is also to identify, investigate, counsel, and screen other family members who may be at risk of carrying the gene for the condition; this role is exemplified in the case of VHL disease. The geneticist may act as a coordinator for the various screening tests, including pre- symptomatic molecular diagnosis, and will provide continuity of care to the families. In addition, (s)he will be able to offer both disease counselling and genetic counselling which will help prospective parents to make informed choices about decisions to undertake predictive testing and on reproductive issues. They will also keep families informed of recent advances in the condition. However, in order for the register to be able to fulfil this role, increased awareness is needed among neurosurgeons and other clinicians dealing with potential cases, so that appropriate referrals are made for ongoing screening.

1 Melmon KL, Rosen SW. Lindau's disease. Review of the literature and study of a large kindred. $\mathrm{Am} \mathcal{F} \mathrm{Med} 1964$ 36:595-617

2 Lamiell JM, Salazar FG, Hsia YE. Von Hippel-Lindau disease affecting 43 members of a single kindred. Medicine (Baltimore) 1989;68:1-29.

3 Maher ER, Yates JRW, Harries R, et al. Clinical features and natural history of von Hippel-Lindau disease. $Q \mathcal{F}$ Med 1990;77:1151-63.

4 Maher ER, Moore AT. Von Hippel-Lindau disease. $B r f$ Ophthalmol 1992;76:743-5.

5 Seizinger BR, Rouleau GA, Ozelius LJ, et al. Von HippelLindau disease maps to the region of chromosome 3 Lindau disease maps to the region of chromosome 3 associat

6 Maher ER, Bentley E, Payne SJ, et al. Presymptomatic diagnosis of von Hippel-Lindau disease with flanking DNA markers. $\mathcal{f}$ Med Genet 1992;29:902-5.

7 Crossey PA, Maher ER, Jones MH, et al. Genetic linkage between Von Hippel-Lindau disease and three microsatellite polymorphisms refines the localisation of the VHL locus. Hum Molec Genet 1993;2:279-82.

8 Latif F, Tory K, Gnarra J, et al. Identification of the von Hippel-Lindau disease tumour suppressor gene. Science 1993;260:1317-20.

9 Harries RWJ. A rational approach to radiological screening in von Hippel-Lindau disease. $₹$ Med Screening 1994;1: $88-95$.

10 Jennings AM, Smith C, Cole DR, et al. Von Hippel-Lindau disease in a large British family: clinicopathological features and recommendations for screening and follow-up. $Q \mathcal{H}$ Med 1988;66:233-49.

11 Martz CH. Von Hippel-Lindau disease: a genetic condition predisposing tumour formation. Oncology Nursing Forum 1991;18:545-51.

12 Littler $M$, Harper PS. A regional register for inherited cancers. BMF 1989;298:1689-91.

13 Huson SM, Harper PS, Hourihan MD, Cole G, Weeks RD, Compston DAS. Cerebellar haemangioblastoma and von Hippel-Lindau disease. Brain 1986;109:1297-310.

14 Maher ER, Bentley E, Yates JRW, et al. Mapping of von Hippel-Lindau disease to chromosome $3 p$ confirmed by Hippel-Lindau disease to chromosome 3p confirmed
genetic linkage analysis. $f$ Neurol Sci 1990;100:27-30.

15 Richards FM, Crossey PA, Phipps ME, et al. Detailed mapping of germline deletions of the von Hippel-Lindau disease tumour suppressor gene. Hum Molec Genet 1994, 3:595-8.

16 Crossey PA, Richards FM, Foster K, et al. Identification of intragenic mutations in the von Hippel-Lindau disease tumour suppressor gene and correlation with disease phenotype. Hum Molec Genet 1994;3:1303-8.

17 Maher ER, Iselius L, Yates JRW, et al. Von Hippel-Lindau disease: a genetic study. $\mathcal{Y}$ Med Genet 1991;28:443-7.

18 Office of Population Censuses and Surveys. Vital Statistics, 1993. London: HMSO, 1994

19 Neumann HPH, Wiestler OD. Clustering of features of von Hippel-Lindau syndrome: evidence for a complex genetic Hippel-Lindau syndrome: evidenc

20 Horton WA, Wong V, Eldridge R. Von Hippel-Lindau disease: clinical and pathological manifestations in nine familease: clinical and pathological manifestations in nine famil-
ies with 50 affected members. Arch Intern Med 1976;136: 769-77.

21 Davies DR, Norman AM, Whitehouse RW, Evans DGR Non-expression of von Hippel-Lindau phenotype in an obligate carrier. Clin Genet 1994;45:104-6.

22 Tada S, Yamagishi J, Kobayashi H, Hata Y, Kobari T. The incidence of simple renal cyst by computed tomography. Clin Radiol 1983;34:437-9. 
Appendix Screening protocol used at St Mary's Hospital, Manchester

Initial screening for an affected person or at risk relative

(1) Full clinical examination.

(2) 24 hour urine collection for VMAs and normetadrenaline.

(3) Fundoscopy if aged 5 years or over.

(4) Upper abdominal USS if aged 16 years or over.

(5) MR head scan* if aged 16 years or over.

Patients are then categorised as either "affected" or "unaffected" but at risk, and are followed up as detailed below.

Follow up screening of "unaffected" family members

(1) Annual full clinical examination.

(2) Annual 24 hour urine collection for VMAs and normetadrenaline.

(3) Annual fundoscopy if aged 5 years or over.

(4) Annual upper abdominal USS if aged 16 years or over.

If renal cysts develop, then US should be supplemented with MR scans* of the upper abdomen (including pancreas, kidneys, and adrenal glands).

(5) MR head scan* recommended only following development of focal neurological signs (either symptomatic or detected by clinical screen).

Follow up of "affected" family members

As for follow up of "unaffected" family members with the following additional considerations.

(1) Early recourse to abdominal CT/MR scans* may be appropriate if there are suspicious signs on the US scan.

(2) Tailoring of the follow up investigations to suit the pattern of disease in particular affected subjects and families may be appropriate, for example, annual MR head scan* may be appropriate to monitor an asymptomatic CHB.

Recurrent radiological examinations (for example, intravenous pyelograms) outside those proposed are not undertaken so that unnecessary and unhelpful radiation exposure is avoided.

* Until recently, CT scans have been used. 